\title{
Long axial range swept-source OCT instrument enhanced by Complex Master-Slave processing
}

\author{
Manuel J. Marques ${ }^{\mathrm{a},}$, Adrian Bradu ${ }^{\mathrm{a}}$, Sylvain Rivet ${ }^{\mathrm{b}}$, and Adrian Podoleanu ${ }^{\mathrm{a}}$ \\ aApplied Optics Group, School of Physical Sciences, University of Kent, Canterbury CT2 \\ 7NH, United Kingdom. \\ ${ }^{\text {b}}$ Laboratoire d'optique et de magnétisme EA938, IBSAM, Université de Bretagne \\ Occidentale, 6 avenue Le Gorgeu, C.S. 93837, 29238 Brest Cedex 3, France. \\ *M. J . Marques@kent.ac.uk
}

\begin{abstract}
We report on the use of the Complex Master-Slave (CMS) method to obtain a long axial range in a sweptsource OCT system, well above the axial range limit imposed by the $k$-clock of the optical source. This is achieved without the need for software-based $k$-domain re-sampling or employing an additional Mach-Zehnder interferometer providing a stable $k$-clock signal to the digitizer board. An imaging range of over $17 \mathrm{~mm}$ is reported in each case using a commercially available swept source from either Axsun and Santec operating in the $1 \mu \mathrm{m}$ region, with a $100 \mathrm{kHz}$ repetition rate, which is about three times the range achievable using either source's built-in $k$-clock. We have also analyzed the impact the digitization has on the axial range and resolution of the system.
\end{abstract}

\section{INTRODUCTION}

In frequency-domain optical coherence tomography (FD-OCT) methods such as spectral-domain (SD-OCT) and swept-source OCT (SS-OCT), the axial range attainable is limited by the maximum frequency sampling rate used to digitize the interferometric spectra. The instantaneous coherence length of swept sources is generally much longer than the coherence length of the diffracted waves after diffraction in spectrometers, therefore SSOCT systems exhibit a longer axial range than their SD-OCT counterparts. An axial range exceeding meters was demonstrated in a recent report on ultra-long coherence length VCSEL tunable lasers. ${ }^{1}$

These long axial range SS-OCT systems are useful in a number of scenarios, ranging from obtaining vascularization maps of large sections of tissue ${ }^{2,3}$ to depth-encoding several measurements in SS-OCT systems with extended functionality, such as passive, multiple input state PS-OCT ${ }^{4}$ and the BRAD concept, ${ }^{5}$ which allows the sample to be imaged with different orientation angles via a multi-mode optical fiber.

A long coherence length swept source requires multi-GS/s sampling rates. Due to the high cost of digitizers and the requirement to produce a GHz-range clock signal, the majority of reports on SS-OCT are limited to an axial range of $3-5 \mathrm{~mm}$. The clock signal used in the spectral sampling operation is the method of choice to perform re-sampling of the spectra, and effectively this dictates the sampling rate of the spectra acquisition. This ultimately limits the axial range to values that are well below the instantaneous coherence length of the swept source.

In order to attain longer axial ranges, strategies bypassing the source's built-in $k$-clock have been reported, such as: (i) using a dedicated Mach-Zehnder interferometer (MZI) with a large optical path difference (OPD) between the MZI arms, coupled with a high-speed photo-detector that produces a clock signal at high frequen$\operatorname{cies}^{4,6,7}$ or (ii) digitizing the interferogram without resorting to an external clock, followed by software-based re-sampling procedures. ${ }^{8}$ These strategies introduce additional complexity and impact the performance of the system. 
It is possible to improve the software-based re-sampling procedures in terms of speed by implementing the algorithms in GPUs or FPGAs; as an alternative approach, one can work with the interferogram in its original domain post-digitization, and modify the kernel of the Fourier transform. This is effectively the basis of the complex Master-Slave (CMS) method reported by our group. ${ }^{9}$ It has been shown that the CMS method presents performance gains when compared to the conventional procedure of software-based re-sampling followed by FFT.

In this communication, we report on the possibility of utilizing the whole axial imaging range provided by the instantaneous coherence length of the swept source, using the CMS approach. This is demonstrated here on two commercially available swept source lasers operating in the 1-micron region. By not utilizing the $k$-clock function and maximizing the sampling rate of the electronic digitizer, we demonstrate up to $17 \mathrm{~mm}$ axial imaging range, well above the $3.7 \mathrm{~mm}^{10}$ and $4.0 \mathrm{~mm}^{11}$ figures imposed by the sources' $k$-clock. Since CMS is employed, no dispersion compensation or additional signal conditioning is necessary, as demonstrated in previous publications. ${ }^{9,12,13}$ To compare our approach against a typical usage scenario employing the same optical sources while utilizing the clock supplied by the manufacturer, we have also characterized the system in that configuration.

\section{EXPERIMENTAL SET-UP}

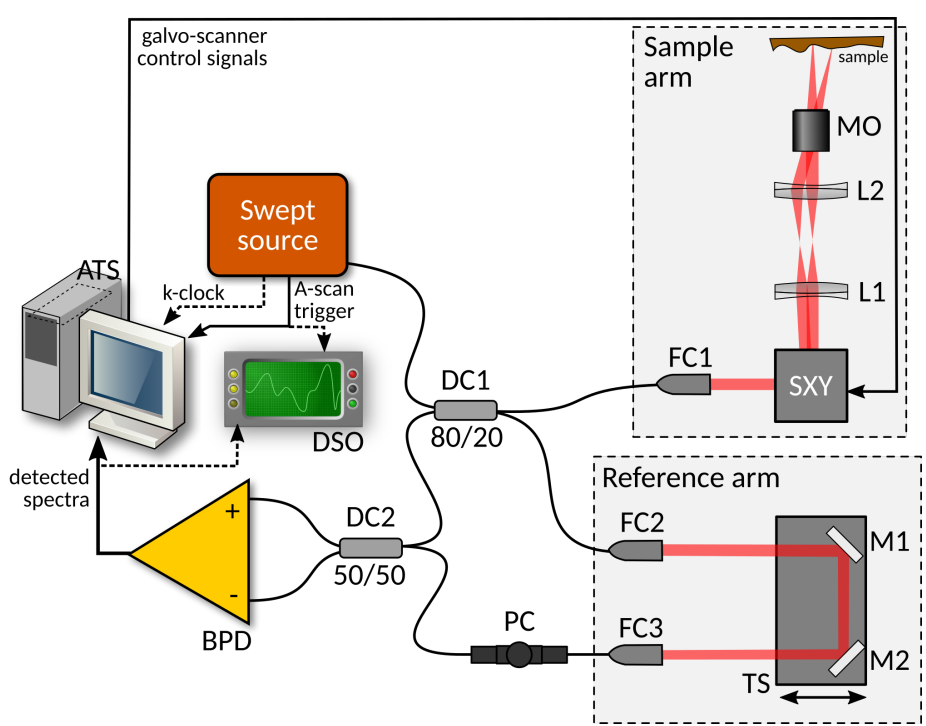

Figure 1. Experimental set-up used to demonstrate the long axial range CMS procedure. DC1-2: fiber-based directional couplers; FC1-3: fiber collimators; SXY: pair of galvo-scanners (Cambridge Technologies, model 6110); L1-2: achromatic lenses forming a telecentric configuration; MO: microscope objective; M1-2: flat protected silver mirrors; BPD: balanced photo-detector; PC: fiber-based inline polarization controller; TS: translation stage; ATS: AlazarTech ATS9360 digitizer board (inside computer); DSO: digital storage oscilloscope.

The experimental set-up is based on a Mach-Zehnder interferometer with balanced detection, which has been described elsewhere, ${ }^{9,14}$ with a schematic representation presented in Fig. 1. The optical interferometer is illuminated with light from a swept source $(100 \mathrm{kHz}$ A-line rate, $1.06 \mu \mathrm{m}$ central wavelength; either Axsun Technologies, Billerica, MA, USA or Santec HSL-10100, Aichi, Japan), and the resulting channeled spectra (CS) are detected using a balanced photo-detector, BPD (Thorlabs PDB481C-AC, 1.0 GHz, optimized for $1.06 \mu \mathrm{m}$ ) followed by electrical high-pass filtering (Thorlabs EF515, > $10 \mathrm{MHz}$ passband) and acquisition by either a high-speed digitizer board, ATS (AlazarTech ATS9360, up to $1.8 \mathrm{GS} / \mathrm{s}$ sampling rate, 800MHz A/D clock rate) or by a $1 \mathrm{GHz}$ digital storage oscilloscope, DSO (LeCroy WaveRunner $104 \mathrm{MXi}$, up to $10 \mathrm{GS} / \mathrm{s}$ 

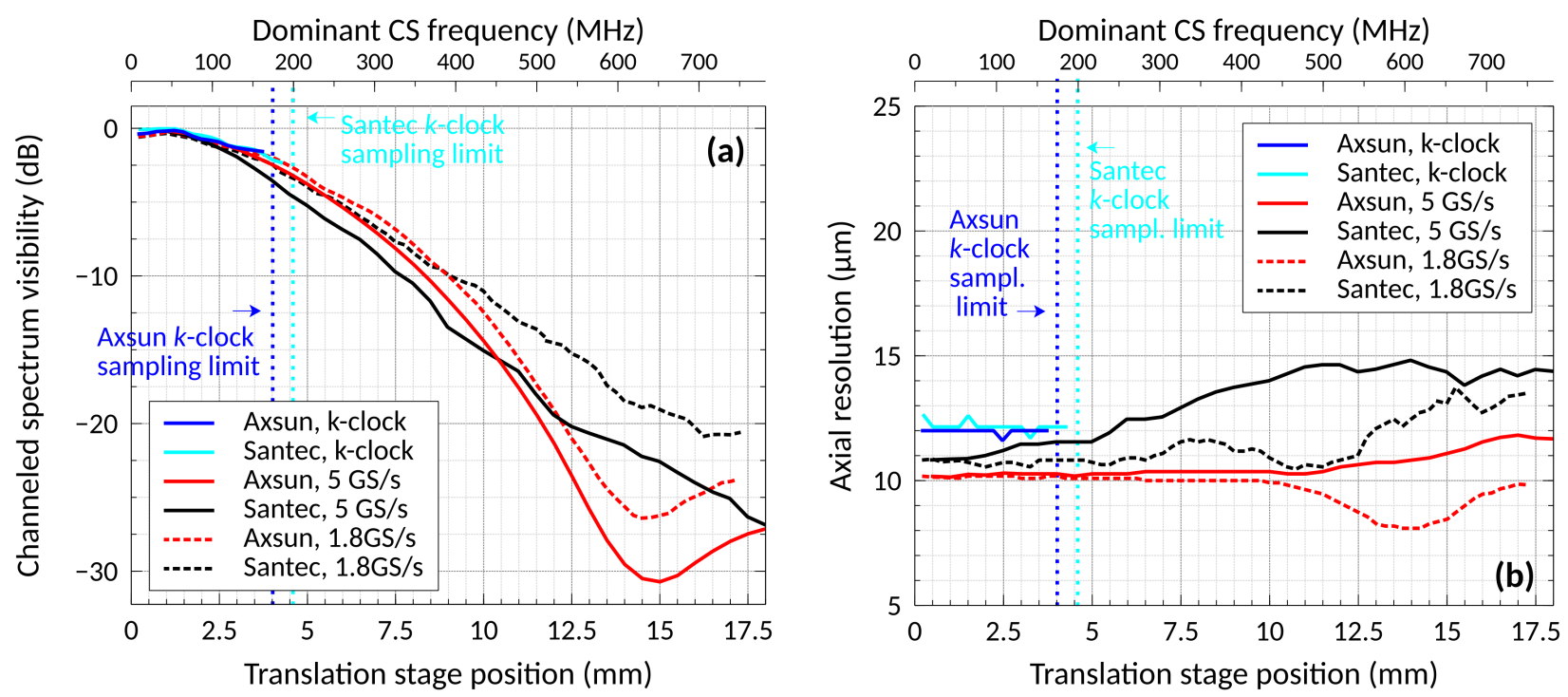

Figure 2. (a) CS visibility versus TS position, obtained from data sets acquired with either the DSO at 5 GS/s (dashed lines) or the ATS at $1.8 \mathrm{GS} / \mathrm{s}$ (continuous lines); red (maximum sampling rate, no $k$-clock) and dark blue ( $k$-clock) traces correspond to the Axsun source, whereas black (maximum sampling rate, no $k$-clock) and cyan ( $k$-clock) traces correspond to the Santec; vertical traces represent the sampling limits when the Axsun (dark blue) or the Santec (cyan) $k$-clock are used. (b) Axial resolution estimated from the A-scan peak FWHM for the same cases described in (a). Top scale represents the dominant (peak) frequency of the CS for each TS position, obtained using a FFT-based analysis.

sampling rate, $1 \mathrm{GHz}$ input bandwidth). For part of this study, the source $k$-clock was used as the sampling clock for the ATS digitizer board.

In order to fully characterize the sensitivity drop-off with depth, a flat silver mirror was placed in the focal plane of the objective lens MO in the object arm of the interferometer, with no lateral scanning applied. The optical path difference (OPD) was adjusted by using a translation stage (TS) that varies the reference arm length.

\section{RESULTS AND DISCUSSION}

The spectra were processed using the CMS method, as described in Rivet et al. ${ }^{12}$ For each CS acquired, the corresponding peak of the A-scan was located and its amplitude represented against the TS position, which can be linked to an OPD value. The full data sets for the two sources, acquired with either the ATS (with and without the source $k$-clock being used) or the DSO, are represented in Fig. 2. In sub-figure (a) the CS visibility versus TS position is presented, while in sub-figure (b) the axial resolution (estimated from the A-scan peak width) versus TS position is presented.

When the $k$-clock from the optical source is engaged during data acquisition, the imaging depth is significantly reduced. For the Axsun source, with a maximum $k$-clock frequency of $350 \mathrm{MHz}$, the maximum imaging range is observed at $3.7 \mathrm{~mm}$. The $k$-clock for the Santec source has a slightly higher maximum frequency, at $400 \mathrm{MHz}$, which increases the maximum imaging range to just over $4.0 \mathrm{~mm}$. In Fig. 2 the dark blue vertical line represents the axial range limit introduced by the $k$-clock of the Axsun source; due to the $350 \mathrm{MHz}$ sampling frequency, the maximum dominant CS frequency which can be sampled in this case is $175 \mathrm{MHz}$. For the Santec, that limit (depicted by the vertical cyan line) is slightly raised to $200 \mathrm{MHz}$.

In either case, the drop in the visibility profile at the maximum imaging range is small (under $-2.5 \mathrm{~dB}$ ), which is aided by the long coherence length reported by the manufacturers of these sources. ${ }^{10,11}$ Figure $2(\mathrm{a})$ 
also contains the CS visibility obtained for the spectra acquired with and without the source's $k$-clock, in the latter case using digital sampling rates of either $1.8 \mathrm{GS} / \mathrm{s}$ (acquired with the ATS) or $5 \mathrm{GS} / \mathrm{s}$ (acquired with the DSO).

By having a larger digital sampling rate $(5 \mathrm{GS} / \mathrm{s}$ ), the DSO would be expected to reach longer imaging depths than the ATS. Despite that, for the same optical source the curve corresponding to the ATS exhibits higher visibility than that of the DSO for the same OPD. This may be due to the limited bit depth resolution of the DSO (8-bit) versus the 12-bit depth of the ATS.

With both the DSO and ATS we are able to reach over $17 \mathrm{~mm}$ imaging range, as shown in Fig. 2 (a). However, some care must be taken in the interpretation of these results, as the cut-off frequencies for the balanced photo-detector, ATS and DSO are, respectively, $1 \mathrm{GHz}, 800 \mathrm{MHz}$ and $1 \mathrm{GHz}$. By performing a frequency-based analysis on the raw CS obtained for different OPDs it was found ${ }^{14}$ that a TS position of $18 \mathrm{~mm}$ yields a maximum CS frequency of $800 \mathrm{MHz}$, therefore one is to expect not just attenuation but also a degradation of the axial resolution at higher imaging ranges, simply because the higher CS frequencies will be further attenuated.

The behavior is well illustrated in Fig. 2 (b), with the A-scan peak width increasing in most curves with increasing TS position, even if the axial resolution does not decrease by more than $50 \%$ in relation to the $10 \mu \mathrm{m}$ baseline throughout the whole measurement range. It appears that the resolution degradation with increasing TS position is higher for the Santec than for the Axsun, which we conjecture to be related with increasing chirping in the CS due to tuning non-linearities - for the same TS position, the corresponding CS measured with the Santec has a frequency spread nearly twice as large ${ }^{14}$ as that measured with the Axsun.

The resolution degradation seems to be more pronounced with the DSO, too; we again attribute this to the limited bit-depth of this device compared to the ATS, as at large OPDs the noise floor is within range of the signal level, with the effect being exacerbated by the limited number of digitization levels.

The data sets acquired using the source $k$-clock appear to have worse resolution figures, however this can be explained from different sampling lengths employed for the acquisition of these data sets; the digitized spectra do not cover exactly the same tuning range of the source, with the $k$-clock cases covering a marginally shorter spectral range interval, which in turn yields a slightly worse axial resolution.

In Fig. 3 two composite images are presented, resulting from the fusion of several B-scans of a retinal phantom (Rowe Technical Design OCT Model Eye, Orange County, CA, USA) taken at different reference arm length settings, using the Axsun swept source. With the $k$-clock on, the imaging range is restricted to just under $4 \mathrm{~mm}$, matching the blue vertical line in Fig. 2 (a). Without resorting to the $k$-clock and employing a sampling rate of $1.8 \mathrm{GS} / \mathrm{s}$, it is possible to still obtain a meaningful image at depths close to $16 \mathrm{~mm}$, albeit with a sensitivity penalty, which matches the CS visibility figures represented in Fig. 2 (a).

In conclusion, we have demonstrated ${ }^{14}$ that the MS-OCT processing, when coupled with a high-throughput digitizer card is capable of achieving an imaging range close to the source's imaging range, since it bypasses the limitations introduced by the source's built-in $k$-clock. The limitations shown by the results presented in this communication stem from the limited sampling rate of the equipment used and not by the method itself.

The enhancement proposed in this communication could help simplifying OCT applications that essentially rely on a long axial range, such as those mentioned earlier in this communication, ${ }^{2-5}$ or just long axial range imaging applications, such as full corneal imaging. ${ }^{9}$ Depending on the application, this might need to be complemented by using either low NA interface optics in the sample arm or by employing Bessel beams. ${ }^{15,16}$ If high NA interface optics are needed, then this method can be combined with a Gabor fusion strategy ${ }^{17,18}$

\section{ACKNOWLEDGMENTS}

The authors wish to acknowledge the following funding sources: H2020 Marie Skłodowska-Curie Actions (MSCA) (625509), Engineering and Physical Sciences Research Council (EPSRC) ('REBOT', EP/N019229/1), H2020 European Research Council (ERC) ('ADASMART', 754695), National Institute for Health Research 

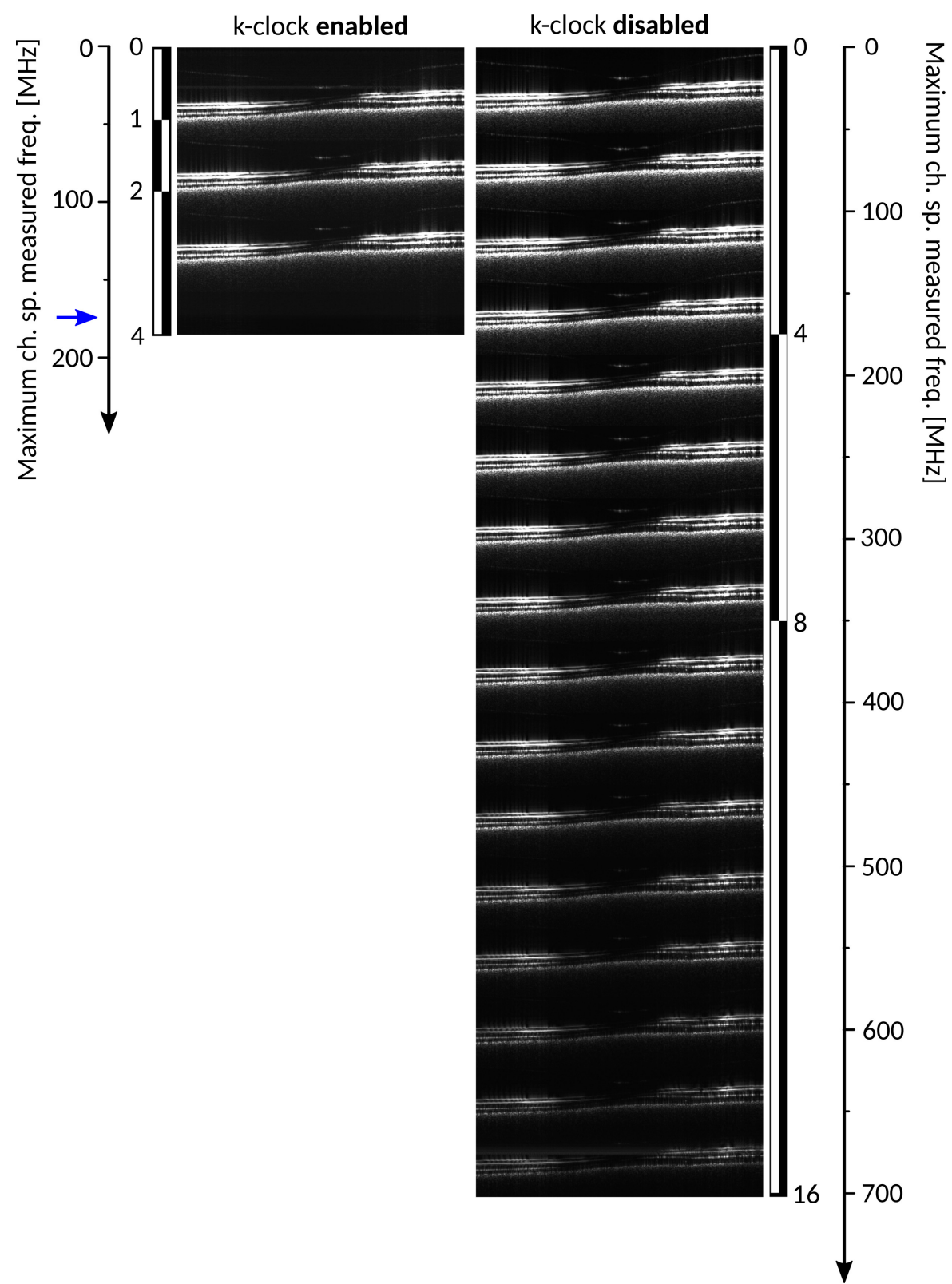

Figure 3. Composite image generated from multiple B-scans taken at different OPD settings (by varying the TS position in steps of $1 \mathrm{~mm}$ ), using the Axsun source. (a) B-scans were acquired while using the source $k$-clock. (b) B-scans were acquired using the ATS clock and with the sampling rate maximized at $1.8 \mathrm{GS} / \mathrm{s}$. Scale bar units: mm. Transversal span is approx. $1 \mathrm{~mm}$. Blue arrow: maximum CS frequency attainable with the $k$-clock of the Axsun source $(175 \mathrm{MHz}$, corresponding to a sampling rate of $350 \mathrm{MHz}$. 
Biomedical Research Centre at Moorfields Eye Hospital NHS Foundation Trust (NIHR), the UCL Institute of Ophthalmology, University College London, and the Royal Society Wolfson research merit award.

\section{REFERENCES}

[1] Wang, Z., Potsaid, B., Chen, L., Doerr, C., Lee, H.-C., Nielson, T., Jayaraman, V., Cable, A. E., Swanson, E., and Fujimoto, J. G., "Cubic meter volume optical coherence tomography," Optica 3(12), 1496-1503 (2016).

[2] Xu, J., Song, S., Men, S., and Wang, R. K., "Long ranging swept-source optical coherence tomographybased angiography outperforms its spectral-domain counterpart in imaging human skin microcirculations," J. Biomed. Opt. 22(11), 116007 (2017).

[3] Xu, J., Song, S., Wei, W., and Wang, R. K., "Wide field and highly sensitive angiography based on optical coherence tomography with akinetic swept source," Biomed. Opt. Express 8(1), 420-435 (2017).

[4] Wang, Z., Lee, H.-C., Ahsen, O. O., Lee, B., Choi, W., Potsaid, B., Liu, J., Jayaraman, V., Cable, A., Kraus, M. F., Liang, K., Hornegger, J., and Fujimoto, J. G., "Depth-encoded all-fiber swept source polarization sensitive OCT," Biomed. Opt. Express 5, 2931-2949 (Sep 2014).

[5] Eugui, P., Lichtenegger, A., Augustin, M., Harper, D. J., Muck, M., Roetzer, T., Wartak, A., Konegger, T., Widhalm, G., Hitzenberger, C. K., Woehrer, A., and Baumann, B., "Beyond backscattering: optical neuroimaging by BRAD," Biomed. Opt. Express 9, 2476-2494 (Jun 2018).

[6] Johnson, B., Atia, W., Kuznetsov, M., Cook, C., Goldberg, B., Wells, B., Larson, N., McKenzie, E., Melendez, C., Mallon, E., Woo, S., Murdza, R., Whitney, P., and Flanders, D., [Optical Coherence Tomography: Technology and Applications], ch. 21, p. 639, Springer, 2 ed. (2015).

[7] Liang, K., Ahsen, O. O., Wang, Z., Lee, H.-C., Liang, W., Potsaid, B. M., Tsai, T.-H., Giacomelli, M. G., Jayaraman, V., Mashimo, H., et al., "Endoscopic forward-viewing optical coherence tomography and angiography with mhz swept source," Optics letters 42(16), 3193-3196 (2017).

[8] Makita, S., Fabritius, T., and Yasuno, Y., "Full-range, high-speed, high-resolution 1- $\mu$ m spectral-domain optical coherence tomography using BM-scan for volumetric imaging of the human posterior eye," Optics express 16(12), 8406-8420 (2008).

[9] Bradu, A., Rivet, S., and Podoleanu, A., "Master/slave interferometry-ideal tool for coherence revival swept source optical coherence tomography," Biomed. Opt. Express 7(7), 2453-2468 (2016).

[10] Axsun Technologies, "Swept lasers for OCT: 1060 \& $1310 \mathrm{~nm}$ high speed swept laser engines." Online. Last checked: 22 Sept 2017.

[11] Santec Corporation, "MEMS Based Swept Source HSL-10/20." Last accessed: 23 Jul 2018.

[12] Rivet, S., Maria, M., Bradu, A., Feuchter, T., Leick, L., and Podoleanu, A., "Complex master slave interferometry," Opt. Express 24(3), 2885-2904 (2016).

[13] Bradu, A., Maria, M., and Podoleanu, A. G., "Demonstration of tolerance to dispersion of master/slave interferometry," Opt. Express 23, 14148-14161 (Jun 2015).

[14] Marques, M. J., Rivet, S., Bradu, A., and Podoleanu, A., "Complex master-slave for long axial range swept-source optical coherence tomography," OSA Continuum 1(4), 1251-1259 (2018).

[15] Ding, Z., Ren, H., Zhao, Y., Nelson, J. S., and Chen, Z., "High-resolution optical coherence tomography over a large depth range with an axicon lens," Optics Letters 27(4), 243-245 (2002).

[16] Leitgeb, R., Villiger, M., Bachmann, A., Steinmann, L., and Lasser, T., "Extended focus depth for fourier domain optical coherence microscopy," Optics letters 31(16), 2450-2452 (2006).

[17] Rolland, J. P., Meemon, P., Murali, S., Thompson, K. P., and Lee, K.-s., "Gabor-based fusion technique for optical coherence microscopy," Opt. Express 18(4), 3632-3642 (2010).

[18] Cernat, R., Bradu, A., Israelsen, N. M., Bang, O., Rivet, S., Keane, P. A., Heath, D.-G., Rajendram, R., and Podoleanu, A., "Gabor fusion master slave optical coherence tomography," Biomed. Opt. Express 8(2), 813-827 (2017). 